\title{
Communication
}

[Comunicação]

\section{Polymorphisms of the coding region of Slc11a1 (Nramp1) gene associated to natural resistance against bovine brucellosis}

\author{
[Polimorfismos na região codificadora do gene Slc1lal (Nramp1) associados à resistência natural \\ contra brucelose bovina] \\ T.A. Paixão ${ }^{1}$, R. Martinez ${ }^{2}$, R.L. Santos ${ }^{3}$ \\ ${ }^{1}$ Instituto de Ciências Biológicas - Universidade Federal de Minas Gerais - Belo Horizonte, MG \\ ${ }^{2}$ Corporación Colombiana de Investigación Agropecuaria - CORPOICA - Bogotá DC, Colombia \\ ${ }^{3}$ Escola de Veterinária - Universidade Federal de Minas Gerais - Belo Horizonte, MG
}

Bovine brucellosis caused by Brucella abortus is an important zoonotic disease characterized by abortion during the last trimester of pregnancy, which results in impaired fertility and decreased milk production in cows (Carvalho Neta et al., 2009). In order to select resistant cattle, identification of genes associated with natural resistance against brucellosis has been extensively investigated. In cattle, natural resistance against $B$. abortus has been linked to polymorphisms within the 3 ' untranslated region (3'UTR) (GT)n microsatellite of the Nrampl gene, which are detectable by single-strand conformational analysis (SSCA) (Feng et al., 1996; Adams et al., 1998; Martinez et al., 2008b). The Nrampl gene, renamed Slcllal, encodes a transmembrane transporter of divalent cation localized in the phagolysosome, which contributes to bactericide activity of macrophage (Wyllie et al., 2002). However, association of this polymorphism to natural resistance against brucellosis was not detected in naturally or experimentally infected cows in Brazil (Paixão et al., 2007), and similar findings were observed by Kumar et al. (2005).

Ables et al. (2002) described other polymorphisms in Slc11A in cattle. However, their influence on natural resistance has been not investigated. Additionally, Martinez et al. (2008a) identified novel polymorphisms in exons of the Slc11Al gene, based on GenBank accession number AC149748, and on information previously reported by Coussens et al. (2004). In this study, were three novel polymorphisms of bovine Slc11Al gene, namely p.D321N (SNP4), p.P356A (SNP5) and p.Q542del (SNP6), previously described by Martínez et al., (2008a), were genotyped in cows experimentally or naturally infected with $B$. abortus in Brazil. The frequency of each genotype and its association to the resistant or susceptible phenotypes to brucellosis in cows were evaluated.

The resistant phenotype in experimentally infected cows was based on the occurrence of normal parturition in spite of a challenge with a high inoculum of $B$. abortus during gestation. Fifty-nine crossbred heifers ranging from 20 to 30 months of age were used in this experiment as previously described (Poester et al., 2006, Paixão et al., 2007). All heifers were serologically negative for brucellosis before challenge, as assessed by the rose bengal plate agglutination test (RBPAT). The heifers underwent estrous synchronization followed by artificial insemination. Pregnancy was confirmed by ultrasonography at 35 days after insemination. Between six and seven months of pregnancy, the heifers were challenged by conjunctival administration of the virulent $B$. abortus strain 2308. Each heifer was inoculated with $50 \mu \mathrm{L}$ in each eye (total of $100 \mu \mathrm{L}$ per heifer) of a bacterial suspension containing $3.0 \times 10^{8} \mathrm{CFU} / \mathrm{mL}$. They were then observed permanently until abortion or calving. This experimental protocol was approved by the local Committee for Ethical Use

Recebido em 6 de março de 2012

Aceito em 1 de agosto de 2012

E-mail: tatianepaixao@icb.ufmg.br 
of Experimental Animals (CETEA-UFMG, protocol 028/05). Cows were euthanized by electrocution after intravenous administration of 15 to $20 \mathrm{mg} / \mathrm{kg}$ of body weight of xylazine (Coopazine, Coopers, Brazil), and then necropsied within 48 hours after abortion or parturition. Fragments of liver were collected from cows and stored at $-20^{\circ} \mathrm{C}$ for subsequent DNA extraction by guanidinium thiocyanate protocol (Paixão et al., 2007).

A crossbred herd with high rate of abortion and a high seroprevalence of B. abortus (>50\%) was used in this study. No records of vaccination against brucellosis were available for this herd. One hundred lactating cows were selected for collection of blood and serum. Serum samples were analyzed for detection of anti-B. abortus antibodies by RBPAT, standard tube agglutination test (STAT) and 2-mercaptoethanol (2-ME) test. Results were expressed as positive or negative for the RBPAT, and complete agglutination at a dilution of 1:25 or higher was considered positive by 2-ME test. Resistant phenotype in naturally infected cows was based on negative serological results to Brucella diagnostic test (AAT and 2M) since all cows in the herd had been exposed to a high challenge considering the large number of cows serologically positive, with reproductive history of abortion due to $B$. abortus confirmed by bacteriology of vaginal swab. Blood sample collected in EDTA tubes were used to DNA extraction. DNA from white blood cells was extracted using the guanidinium thiocyanate protocol (Paixão et al., 2007).

DNA samples were subjected to genotyping by PCR to detect three new polimorphisms of coding regions Nrampl using primers: SNP4-F 5'GGCTTGGAGGTCTGATTTTC and SNP4-R 5'CGTTGGCTTGCTTACTCCTT, $\begin{array}{llll}\text { product of } & 176 \quad \mathrm{bp} ; & \mathrm{SNP} 5-\mathrm{F}\end{array}$
5'CAAGGAGTAAGCAAGCCAAC and SNP5-R 5'GCTGCCTTAAGGATCAAGGA, product of 350 bp; and SNP6-F 5'TTCCTGTATGGGCTTCCTG and SNP6-R 5'CTTGCTGCCTTCACACACAT, product of $158 \mathrm{bp}$. PCR amplification was performed using Taq DNA Polymerase (Invitrogen, USA), with the following parameters: an initial incubation at $94^{\circ} \mathrm{C}$ for $5 \mathrm{~min}$, followed by 33 cycles of $95^{\circ} \mathrm{C}$ for $1 \mathrm{~min}$, $56^{\circ} \mathrm{C}$ for $1 \mathrm{~min}$ and $72^{\circ} \mathrm{C}$ for $1 \mathrm{~min}$ and finally 4 min at $72^{\circ} \mathrm{C}$. To detect the SNP (single nucleotide polymorphism), 20-100ng of the amplicons were directly subjected to SSCA analysis by mixing the amplicons (v/v) with denaturing $2 \mathrm{X}$ SSCA loading buffer $(95 \%$ formamide, $0.6 \%$ bromophenol blue and $0.6 \%$ xylene cyanole), heating at $94^{\circ} \mathrm{C}$ for $5 \mathrm{~min}$, snap chilling on ice and loading onto 29:1 acrylamide:bis-acrylamide $8 \%$ polyacrylamide gels prepared with TBE buffer. Electrophoresis was allowed to run at $10^{\circ} \mathrm{C}$ and $4 \mathrm{~W}$ for 15 hours and gels were then silver stained (Barroso et al., 1997).

The frequencies of polymorphisms of bovine Slcl1Al with a resistant or susceptible phenotype in experimental or natural infections with $B$. abortus are summarized in Tables 1 and 2 . The homozygous genotypes CC from SPN4 and AA from SPN5 were more frequent in susceptible naturally infected cows. These results indicate a link between Slc11Al genotype and actual resistance, which supports the notion that Slc11Al genotyping may eventually provide an approach for genetic selection of brucellosisresistant cattle. Although there are still controversial results regarding influence of Slc11A1 alleles on natural resistance against brucellosis (Paixão et al., 2007; Martinez et al., 2008 b), this study is a significant contribution towards the development of a protocol for selection of genetically resistant cattle.

Tabela 1. Frequencies of Slc11A1 genotypes in cows experimentally infected with Brucella abortus

\begin{tabular}{|c|c|c|c|c|c|c|c|c|c|c|c|c|}
\hline \multirow{3}{*}{ Phenotypes } & \multicolumn{12}{|c|}{ Genotypes } \\
\hline & \multicolumn{3}{|c|}{ SPN4 $(n=59)$} & \multicolumn{3}{|c|}{ SPN5 $(n=48)$} & \multicolumn{6}{|c|}{ SPN6 $(n=59)$} \\
\hline & $\begin{array}{l}\mathrm{CC} \\
(\%)\end{array}$ & $\begin{array}{l}\text { CT } \\
(\%)\end{array}$ & $\begin{array}{l}\text { TT } \\
(\%)\end{array}$ & $\begin{array}{l}\text { AA } \\
(\%)\end{array}$ & $\begin{array}{l}\mathrm{AB} \\
(\%)\end{array}$ & $\begin{array}{l}\text { BB } \\
(\%)\end{array}$ & $\begin{array}{l}\text { AA } \\
(\%)\end{array}$ & $\begin{array}{l}\mathrm{AB} \\
(\%)\end{array}$ & $\begin{array}{l}\text { BB } \\
(\%)\end{array}$ & $\begin{array}{l}\mathrm{CC} \\
(\%)\end{array}$ & $\begin{array}{l}\mathrm{AC} \\
(\%)\end{array}$ & $\begin{array}{l}\mathrm{BC} \\
(\%)\end{array}$ \\
\hline Resistant $^{1}$ & $\begin{array}{c}8 / 43 \\
(18.6)\end{array}$ & $\begin{array}{l}21 / 43 \\
(48.8)\end{array}$ & $\begin{array}{l}14 / 43 \\
(32.6)\end{array}$ & $\begin{array}{l}21 / 42 \\
(50.0)\end{array}$ & $\begin{array}{c}14 / 42 \\
(33.3) \mathrm{a}\end{array}$ & $\begin{array}{c}7 / 42 \\
(16.7)\end{array}$ & $\begin{array}{l}21 / 43 \\
(48.8)\end{array}$ & $\begin{array}{l}/ 43 \\
(9.3)\end{array}$ & $\begin{array}{l}1 / 43 \\
(2.3)\end{array}$ & $\begin{array}{c}/ 43 \\
(16.3)\end{array}$ & $\begin{array}{c}7 / 43 \\
(16.3)\end{array}$ & $\begin{array}{l}3 / 43 \\
(7.0)\end{array}$ \\
\hline Susceptible $^{2}$ & $\begin{array}{c}3 / 16 \\
(18.7)\end{array}$ & $\begin{array}{c}5 / 16 \\
5 / 16 \\
(31.3) \\
\end{array}$ & $\begin{array}{c}8 / 16 \\
(50) \\
\end{array}$ & $\begin{array}{l}10 / 16 \\
(62.5)\end{array}$ & $\begin{array}{c}1 / 16 \\
(6.2) b^{*}\end{array}$ & $\begin{array}{c}5 / 16 \\
(31.3)\end{array}$ & $\begin{array}{l}10 / 16 \\
(62.5)\end{array}$ & $\begin{array}{c}0 / 16 \\
(0)\end{array}$ & $\begin{array}{c}2 / 16 \\
(12.5) \\
\end{array}$ & $\begin{array}{l}4 / 16 \\
(25) \\
\end{array}$ & $\begin{array}{c}0 / 16 \\
(0)\end{array}$ & $\begin{array}{c}0 / 16 \\
(0)\end{array}$ \\
\hline
\end{tabular}

1. Normal parturition; 2. Abortion. Chi-square test. Fisher's exact test. Values followed by distinct letters in the same column are different. $* \mathrm{P}<0.05$ 
Tabela 2. Frequencies of Slc11A1 genotypes in cows naturally infected with Brucella abortus $(\mathrm{n}=100)$

\begin{tabular}{|c|c|c|c|c|c|c|c|c|c|c|c|c|}
\hline \multirow{3}{*}{ Phenotypes } & \multicolumn{12}{|c|}{ Genotypes } \\
\hline & \multicolumn{3}{|c|}{ SPN4 } & \multicolumn{3}{|c|}{ SPN5 } & \multicolumn{6}{|c|}{ SPN6 } \\
\hline & $\begin{array}{l}\mathrm{CC} \\
(\%) \\
\end{array}$ & $\begin{array}{l}\mathrm{CT} \\
(\%)\end{array}$ & $\begin{array}{l}\text { TT } \\
(\%)\end{array}$ & $\begin{array}{l}\text { AA } \\
(\%)\end{array}$ & $\begin{array}{l}\mathrm{AB} \\
(\%)\end{array}$ & $\begin{array}{l}\text { BB } \\
(\%)\end{array}$ & $\begin{array}{l}\text { AA } \\
(\%)\end{array}$ & $\begin{array}{l}\mathrm{AB} \\
(\%) \\
\end{array}$ & $\begin{array}{l}\text { BB } \\
(\%)\end{array}$ & $\begin{array}{l}\mathrm{CC} \\
(\%)\end{array}$ & $\begin{array}{l}\mathrm{AC} \\
(\%)\end{array}$ & $\begin{array}{l}\mathrm{BC} \\
(\%)\end{array}$ \\
\hline Resistant $^{1}$ & $\begin{array}{c}1 / 35 \\
(2.7) \mathrm{a}\end{array}$ & $\begin{array}{c}9 / 35 \\
(25.7) \mathrm{a}\end{array}$ & $\begin{array}{l}25 / 35 \\
(71.4)\end{array}$ & $\begin{array}{l}16 / 35 \\
(45.7 \mathrm{a}\end{array}$ & $\begin{array}{c}12 / 35 \\
(34.2) \mathrm{a}\end{array}$ & $\begin{array}{c}7 / 35 \\
(20.0) \mathrm{a}\end{array}$ & $\begin{array}{l}26 / 35 \\
(74.3)\end{array}$ & $\begin{array}{l}3 / 35 \\
(8.7)\end{array}$ & $\begin{array}{l}1 / 35 \\
(2.8)\end{array}$ & $\begin{array}{l}1 / 35 \\
(2.8)\end{array}$ & $\begin{array}{c}4 / 35 \\
(11.4)\end{array}$ & $\begin{array}{c}0 / 35 \\
(0)\end{array}$ \\
\hline Susceptible $^{2}$ & $\begin{array}{c}15 / 65 \\
(23.1) b^{* *} \\
\end{array}$ & $\begin{array}{c}4 / 65 \\
(6.1) b^{*}\end{array}$ & $\begin{array}{l}46 / 65 \\
(70.8) \\
\end{array}$ & $\begin{array}{c}52 / 65 \\
(80.0) b^{* * * *} \\
\end{array}$ & $\begin{array}{c}10 / 65 \\
(15.3) b^{*} \\
\end{array}$ & $\begin{array}{c}3 / 65 \\
(4.6) b^{*}\end{array}$ & $\begin{array}{l}46 / 65 \\
(70.8) \\
\end{array}$ & $\begin{array}{l}5 / 65 \\
(7.7) \\
\end{array}$ & $\begin{array}{l}1 / 65 \\
(1.5) \\
\end{array}$ & $\begin{array}{l}2 / 65 \\
(3.1) \\
\end{array}$ & $\begin{array}{l}11 / 65 \\
(16.9) \\
\end{array}$ & $\begin{array}{c}0 / 65 \\
(0) \\
\end{array}$ \\
\hline
\end{tabular}

1. Positive or 2. Negative serology, under conditions of high seroprevalence and high abortion rate in a closed heard. Chi-square test. Fisher's exact test. Different letters in the same column means statistically significant difference $* \mathrm{P}<0.05 * * \mathrm{P}<0.01 * * * \mathrm{P}>0.001$

Keywords: bovine, brucellosis, natural resistance, Nramp1, Slc11a1

\section{RESUMO}

Brucelose bovina causada por Brucella abortus é uma importante doença zoonótica, caracterizada pela ocorrência de aborto durante o último trimestre da gestação, o que resulta em diminuição da fertilidade da produção de leite em vacas. A identificação de genes associados à resistência natural contra brucelose tem sido investigada com o objetivo de selecionar animais resistentes à doença. Em bovinos, é controversa a resistência natural contra $\mathrm{B}$. abortus associada ao polimorfismo da região $3^{\prime}$ UTR do gene Slc11A1 (Nramp1). Polimorfismos localizados na sequência codificadora de Slc11A1 têm sido identificados em bovinos, contudo a influência sobre a resistência natural contra brucelose não é conhecida. No presente estudo, três novos polimorfismos do gene Slc11A1 foram genotipados por análise conformacional de fita simples em vacas experimentalmente ou naturalmente infectadas por B. Abortus, e foram avaliadas a frequência de cada genótipo e sua associação com o fenótipo de resistência ou susceptibilidade à brucelose bovina. Os resultados deste estudo demonstram que alguns genótipos foram mais frequentes em animais considerados fenotipicamente susceptiveis à brucelose.

Palavras-chave: bovino, brucelose, resistência natural, Nramp1, Slc11a1

\section{ACKNOWLEDGEMENTS}

This work was supported by $\mathrm{CNPq}$ (Conselho Nacional de Desenvolvimento Científico e Tecnológico, Brasília, Brazil) and FAPEMIG (Fundação de Amparo a Pesquisa do Estado de Minas Gerais, Belo Horizonte, Brazil). RLS is recipient of a fellowship from $\mathrm{CNPq}$.

\section{REFERENCES}

ABLES, G.P.; NISHIBORI, M.; KANEMAKI, M. et al. Sequence analysis of the NRAMP1 genes from different bovine and buffalo breeds. J. Vet. Med. Sci. v.64, p.1081-1083, 2002.

ADAMS, L.G.; TEMPLETON, J.W. Genetic resistance to bacterial diseases of animals. Rev. Sci. Tech. Off. Int. Epizoot., v.7, p.200-219, 1998.

BARROSO, A.; DUNNER, S.; CANÕN, J. Use of a single strand conformation polymorphism analysis to perform a simple genotyping of bovine k- casein A and B variants. J. Dairy Res., v.64, p.535-540, 1997.
CARVALHO NETA, A.V.; MOL, J.P.; XAVIER, M.N. et al. Pathogenesis of bovine brucellosis. Vet. J., v.184, p.146-155, 2010.

COUSSENS, P.M.; COUSSENS, M.J.; TOOKER, B.C. et al. Structure of the bovine natural resistance associated macrophage protein (NRAMP) gene and identification of a novel polymorphism. DNA Seq., v.15, p.15-25, 2004.

FENG, J.; LI, Y.; HASHAD, M. et al. Bovine natural resistance associated macrophage protein 1 (Nrampl) gene. Genome Res., v.6, p.956-964, 1996.

KUMAR, N.; MITRA, A.; GANGULY, I. et al. Lack of association of brucellosis resistance with (GT)(13) microsatellite allele at 3'UTR of NRAMP1 gene in Indian zebu (Bos indicus) and crossbred (Bos indicus x Bos taurus) cattle. Vet. Microbiol., v.111, p.139-143, 2005. 
MARTÍNEZ, R.; DUNNER, S.; BARRERA, G. Novel variants within the coding regions of the Slc11A1 gene identified in Bos taurus and Bos indicus breeds. J. Anim. Breed Genet., v.125, p.57-62, 2008a.

MARTÍNEZ, R.; TORO, R.; MONTOYA, F. et al. Bovine SLC11A1 3' UTR SSCP genotype evaluated by a macrophage in vitro killing assay employing a Brucella abortus strain. J. Anim. Breed Genet., v.125, p.271-279, 2008 b.

PAIXÃO, T.A.; POESTER, F.P.; CARVALHO NETA, A.V. et al. NRAMP1 3' untranslated region polymorphisms are not associated with natural resistance to Brucella abortus in cattle. Infect. Immun., v.75, p.2493-2499, 2007.
POESTER, F.P.; GONCALVES, V.S.P.; PAIXÃO, T.A. et al. Efficacy of strain RB51 vaccine in heifers against experimental brucellosis. Vaccine, v.24, p.5327-5334, 2006.

WYLLIE, S.; SEU, P.; GOSS, J.A. The natural resistance-associated macrophage protein 1 Slc11a1 (formerly Nramp1) and iron metabolism in macrophages. Microbes Infect., v.4, p.351$359,2002$. 